\title{
CORRIENTES DEL AMBIENTALISMO Y ALTERNATIVAS DE GESTIÓN DESDE LA SUSTENTABILIDAD Y LA ÉTICA AMBIENTAL*
}

Recibido: 24 de mayo de 2013 • Aprobado: 15 de mayo de 2014

\author{
Diana Marcela Sánchez Torres* \\ Marco Aguilera Prado ${ }^{* * *}$
}

\section{RESUMEN}

El presente texto describe las raíces conceptuales de la gestión ambiental y presenta la crítica desde la ética ambiental, para lo cual hace una lectura de los autores representativos. Así, ambientalismo conservacionista, ambientalismo moderado y humanismo crítico se identifican como las corrientes referentes de la gestión ambiental actual, al tiempo que existen otras posturas o visiones alternativas que merecen ser consideradas para el abordaje de la gestión ambiental territorial.

\section{PALABRAS CLAVE}

Sustentabilidad, desarrollo y ambiente.

\section{CLASIFICACIÓN JEL}

Q20, Q56

\section{CONTENIDO}

Introducción; 1. El ambientalismo reconocido de la década de 1970;

2. La crítica desde sustentabilidad y ética ambiental; 3. Gestión, am-

bientalismo y alternativas; 4. A manera de comentario; Bibliografía.

* Artículo de investigación producto del desarrollo del primer objetivo de la tesina "Propuesta de gestión ambiental en el contexto de la sustentabilidad para el espacio público natural urbano de la conurbación Pereira Dosquebradas" realizada en 2012 para optar al título de Magíster en Desarrollo Regional de la Universidad Católica de Pereira.

** Administradora Ambiental de la Universidad Tecnológica de Pereira. Magíster en Gestión del Desarrollo Regional de la Universidad Católica de Pereira. Miembro del Grupo de Investigación en Gestión Ambiental Territorial de la Universidad Tecnológica de Pereira. Catedrático de la Escuela de Administración Pública -ESAP y de la Universidad Tecnológica de Pereira -UTP_. Correo electrónico: dianamarcela. sancheztorres@utp.edu.co, dianamarcela.sancheztorres@gmail.com.

*** Economista de la Universidad Autónoma de Occidente. Mg. Planificación y Administración del Desarrollo Regional de la Universidad de los Andes. Estudiante Doctorado en Economía de la Universidad Nacional Autónoma de México. Profesor Facultad de Ciencias Económicas y Administrativas. Miembro del Grupo de Investigación Desarrollo Empresarial. Universidad Católica de Pereira. Correo electrónico: marco.aguilera@ucp.edu.co 


\section{ENVIRONMENTAL MOVEMENTS AND MANAGEMENT ALTERNATIVES FROM A SUSTAINABILITY AND ENVIRONMENTAL ETHICS SCOPE ABSTRACT}

This paper describes de conceptual roots of environmental management and offers a criticism from an environmental ethics point of view, for which it makes a reading of representative authors. This way, conservative environmentalism, Moderate Environmentalism and Critical Humanism are identified as currents that serve as referents of current environmental management. At the same time other existent postures and alternative visions deserve to be considered for the territorial environmental management approach.

KEY WORDS

Sustainability, development, environment

JEL CLASSIFICATION

Q20, Q56

\section{CONTENT}

Introduction; 1. Recognized environmentalism of the 1970 decade; 2. Criticism from sustainability and environmental ethics; 3. Management, environmentalism and alternatives; 4. As a comment; Bibliography.

\section{CORRENTES DO AMBIENTALISMO E ALTERNATIVAS DE GESTÃO DESDE A SUSTENTABILIDADE E A ÉTICA AMBIENTAL}

\section{RESUMO}

O presente texto descreve as raízes conceituais da gestão ambiental e apresenta a crítica desde a ética ambiental, para o qual faz uma leitura dos autores representativos. Assim, Ambientalismo Conservacionista, Ambientalismo Moderado e Humanismo Crítico se identificam como as correntes referentes da gestão ambiental atual, ao mesmo tempo em que existem outras posturas ou visões alternativas que merecem ser consideradas para a abordagem da gestão ambiental territorial.

\section{PALAVRAS CAHVES}

Sustentabilidade, desenvolvimento e ambiente.

CLASSIFICAÇÃO JEL

Q20, Q56.

\section{CONTEÚDO}

Introdução; 1. O ambientalismo reconhecido da década de 1970; 2. A crítica desde sustentabilidade e ética ambiental; 3. Gestão, ambientalismo e alternativas; 4. A maneira de comentário; Bibliografia. 


\section{INTRODUCCIÓN}

La gestión ambiental evidencia inconsistencias entre las propuestas conceptuales y las praxis, las cuales se hacen visibles en casos como expansiones urbanas en áreas de alta fragilidad ecológica, contaminación de humedales, disposición de residuos sólidos o destrucción de corredores ambientales. Estos problemas son desafíos para las entidades territoriales, debido a la necesidad de brindar soluciones de vivienda o construcción de infraestructura para satisfacer tanto las necesidades de grupos humanos como los intereses de stakeholders y policy-makers que favorecen el crecimiento económico para sostener la plataforma desarrollista y progresista de los territorios (Sánchez, 2013; Cortés y Aguilera, 2012).

La situación pudiera interpretarse como una tensión entre los intereses de los diferentes grupos, que se soluciona con un arreglo político que tiene como resultado la presión sobre el medio físico, toda vez que este no tiene representación o es muy débil. Sin embargo, el asunto trasciende la construcción de ese arreglo como solución a la tensión de los intereses y tiene que ver con la forma en que se entiende el ambiente, lo ambiental y sus relaciones con lo político y lo económico.

De acuerdo con lo anterior, el presente artículo plantea como objetivo precisar en las raíces teóricas de la gestión ambiental como avance en la respuesta a la pregunta: ¿por qué de la perpetuación de los problemas ambientales a pesar de los ya casi 40 años de elaboraciones conceptuales y aplicaciones para la gestión ambiental? Por ello, se empleó el enfoque de la metodología de la investigación holística como forma de abordaje que permite enlazar varios elementos de manera sistémica.

Se identificaron tres corrientes: ambientalismo conservacionista, ambientalismo moderado y humanismo crítico, entre las cuales la segunda es la corriente hegemónica. A pesar de ello, han emergido y coexistido otras conceptualizaciones como el ambientalismo conservacionista, el humanismo crítico y las críticas de sustentabilidad y ética ambiental en el ámbito latinoamericano (Sánchez, 2013).

El presente artículo se divide en cinco secciones que corresponden a (1) El ambientalismo reconocido de la década de 1970; (2) La crítica desde la sustentabilidad y la ética ambiental; (3) Gestión, ambientalismo y alternativas; (4) A manera de comentario; (5) Referencias.

\section{EL AMBIENTALISMO RECONOCIDO DE LA DÉCADA DE 1970}

El origen del ambientalismo, si bien existen raíces filosóficas presocráticas, puede asociarse con la preocupación por el crecimiento de los setenta, enmarcada en la crisis del petróleo y los hallazgos del Club de Roma. Ese ambientalismo se puede 
clasificar en tres corrientes: ambientalismo conservacionista, ambientalismo moderado y humanismo crítico (Pierri, 2005). El ambientalismo moderado es el de mayor aceptación y es la base de la gestión ambiental tradicional mediante una propuesta de crecimiento para la gestión ambiental.

Por su parte, las otras dos corrientes presentan menos acogida, dadas sus posiciones más radicales. Los conservacionistas plantean que el ser humano hace parte de la naturaleza y, por tanto, su relación con la misma debe ser en pie de igualdad y de respeto hacia todas las especies, mientras que el humanismo crítico a través de ecodesarrollo propone alternativas específicas de gestión desde las comunidades, una vez identifican sendas propias de desarrollo en su relación con el entorno, y desde el marxismo plantea la necesidad de un cambio estructural en el modelo de desarrollo que implique nuevas relaciones de producción y el manejo de los medios de producción como manera de construir un proceder diferente en la relación con la naturaleza (Pierri, 2005).

La corriente conservacionista, liderada por biólogos y ecólogos, estuvo expresada por los límites físicos al crecimiento económico y poblacional, y el crecimiento cero. Los límites al crecimiento poblacional tienen raíces en Malthus y los rendimientos decrecientes de la tierra ricardianos son las simientes del límite físico del crecimiento económico. A partir de allí se expone, como posible salida a la presión ambiental y el consecuente riesgo para la vida, el crecimiento cero o el estado estacionario. El primero que abogaba por el freno al crecimiento demográfico, y el segundo, por no producir por encima del crecimiento de la población y sus necesidades, por cuanto existe dependencia de la naturaleza para la satisfacción de esas necesidades y los tiempos de recurrencia (de las necesidades humanas) son más cortos que los de reproducción de la naturaleza (Pierri, 2005).

El informe del Club de Roma en 1972 tuvo gran impacto en esta corriente al ser el de mayor difusión. El informe planteó la necesidad de realizar reducciones en i) la utilización de recursos naturales en un 75 \%; mediante reciclaje, ii) la contaminación en un $50 \%$, iii) las inversiones en un $40 \%$, y iv) la natalidad en un $30 \%$. Posterior a este, la relaboración del equipo Meadows (el World-3) determinó el final del crecimiento antes del año 2100 por tres sendas simultáneas: la sobre explotación del suelo conduce a la erosión, y la producción de alimentos desciende; los recursos son deteriorados por una próspera población mundial, y la contaminación aumenta lo que produce un descenso aún mayor en la producción de alimentos y una elevación de la tasa de mortalidad (Pierri, 2005).

La impronta filosófico-política del conservacionismo es la ecología profunda de Arne Naess y George Sessions. Dentro de sus planteamientos está renovar el 
acercamiento ético-jurídico del ser humano a la naturaleza, al hacer de esta un sujeto de derecho, de modo que la relación hombre-naturaleza debe construirse en términos de simbiosis y reciprocidad, ya que el derecho de dominio y de propiedad se asimila al parasitismo. Asimismo, se plantea buscar no solo el bienestar humano sino extender el reconocimiento de los "fines en sí" más allá de la esfera del hombre (Ferry, 1992).

El ambientalismo moderado se establece como una corriente que pretende hacer compatibles el desarrollo basado en el crecimiento económico y el cuidado de la naturaleza. Sus inicios corresponden a la Cumbre de Medio Humano o Cumbre de Estocolmo realizada por la ONU en 1972 a la cual llegó la postura de poner límites al crecimiento de acuerdo con los postulados del Club de Roma conforme a los modelos elaborados para determinar el futuro del planeta. Sin embargo, el planteamiento de los países en vía de desarrollo sobre la situación de pobreza, que hacía necesario el crecimiento económico para satisfacer las necesidades básicas de la población, puso de manifiesto las diferencias existentes entre estos y los países desarrollados quienes ya tenían sus necesidades satisfechas y, por tanto, podían concentrarse en conservar mientras las condiciones de los países del Tercer Mundo no daban para pensar en conservación. La pobreza se constituyó como elemento determinante de los problemas ambientales y su solución prioritaria frente a la degradación de la naturaleza. De allí que el enfoque de esta corriente es antropocéntrico, puesto que la naturaleza es vista como un stock de materias primas para satisfacer las necesidades de los seres humanos y resolver el problema fundamental que es la pobreza. Sin embargo, no hay cuestionamientos de fondo sobre el modelo de desarrollo, la distribución de la riqueza, las desigualdades sociales, sino que la solución de esta corriente es continuar con el mismo modelo de desarrollo basado en el crecimiento económico con márgenes de conservación, pretendiendo solucionar los problemas humanos y ambientales, donde los últimos son consecuencia de los primeros.

El principal referente enmarcado dentro de la corriente del ambientalismo moderado es el desarrollo sostenible planteado en 1987 por la Comisión Brundtland y visto como aquel que permite satisfacer las necesidades de las generaciones actuales sin comprometer las de las generaciones futuras, lo cual establece que la prioridad es el ser humano, relegando a un segundo plano las otras formas de vida presentes en el planeta Tierra.

Esta postura está dentro del contexto de la economía ambiental que sostiene que la naturaleza puede valorarse de manera económica, es proveedora de bienes públicos, se pueden asignar derechos de propiedad sobre ella, y puede estar sujeta a mecanismos de mercado. De esta manera, costos de oportunidad, disponibilidades 
a pagar, precios sombra como resultado del ejercicio empírico están inmersos en la dinámica del crecimiento económico que envuelve el desarrollo sostenible, de modo que el valor de la naturaleza y su existencia dependen de la valoración humana.

En esa misma dirección, los "Procedimientos de gestión para el desarrollo sustentable", de A. Dourojeanni, el modelo de gestión más difundido, considera que apuntar al desarrollo sustentable es hacer compatibles por lo menos cuatro procesos de gestión: i) materialización de acciones que lleven al crecimiento económico; ii) transacciones, que conduzcan a la equidad; iii) integración de áreas temáticas, que conduzcan al trabajo interdisciplinario; y iv) la incorporación de la temática ambiental (CEPAL, 1994). De nuevo, la condición inicial es el crecimiento económico que favorece la equidad y el cuidado de lo no-humano.

Por último la corriente humanística crítica basa sus principios en la transformación que se debe dar desde la sociedad para contribuir a un cambio en la gestión del patrimonio natural. Esta corriente presenta dos vertientes: la primera desde el ecodesarrollo y la segunda desde la postura marxista. La primera vertiente presenta la necesidad de recuperar los valores comunitarios y saberes ancestrales que reconocen cuál es el manejo que debe darse a la naturaleza para su preservación y la satisfacción de las necesidades básicas de la población. La lógica de este desarrollo ecológico es que debe partir desde las bases de la sociedad, es decir, desde las mismas comunidades y, por tanto, no debe ser un desarrollo impuesto a partir de un modelo hegemónico. De igual forma, dentro del ecodesarrollo se plantea la necesidad de emplear tecnologías apropiadas o tecnologías limpias que no causen daño al ambiente, al igual que se debe tener una planeación regional que reconozca las necesidades de cada contexto para hallar soluciones efectivas. Algunos de los principales exponentes de esta subcorriente son Schumacher, Sachs y Galtung (Pierri, 2005).

La vertiente marxista está representada en el Modelo Mundial Latinoamericano propuesto por la Fundación Bariloche. Este modelo planteaba un cuestionamiento a la propiedad de los medios de producción, ya que esta se reconoció como la causa fundamental del problema ambiental. La propuesta estaba dirigida a la gestión colectiva de los medios de producción para satisfacer las necesidades básicas de la sociedad y, de esta manera, generar las condiciones para un uso adecuado del patrimonio natural. Los insumos empleados por este modelo fueron las variables y los resultados que arrojó el modelo World-3. Sin embargo, se consideraba que la crisis no estaba en el futuro como se pronosticaba en dicho modelo, sino que la crisis ya se daba en el presente con la pobreza y miseria en el mundo, principalmente en los países subdesarrollados, para lo cual hicieron su propuesta de carácter estructural 
frente a los cambios que debían realizarse en el modelo de desarrollo que permitieran lograr los objetivos ambientales, planteando, por lo tanto, una idea de fondo frente al ecodesarrollo y a la corriente del ambientalismo moderado.

\section{LA CRÍTICA DESDE SUSTENTABILIDAD Y ÉTICA AMBIENTAL}

Después de la década de 1980 en medio de los cambios estructurales latinoamericanos a favor del mercado y como respuesta a la hegemonía del neoclasicismo teórico de la economía ambiental como base de la gestión ambiental, han surgido algunas producciones ligadas al reconocimiento antropológico de las relaciones de los humanos en contextos espacio-temporales específicos. Entre esas propuestas se destacan la sustentabilidad como alternativa a la sostenibilidad ligada al sustainable development ${ }^{1}$ y la construcción de una ética ambiental. Esas propuestas tienen como exponentes más representativos a Enrique Leff, Augusto Ángel Maya, Julio Carrizosa y Ana Patricia Noguera (Sánchez, 2013).

La sustentabilidad es la apertura a lo social desde las distintas percepciones, visiones, prácticas e ideologías que reconocen en la naturaleza su valor intrínseco y respetan las interrelaciones que en ella se llevan a cabo. Este planteamiento corresponde a una racionalidad ambiental que difiere de la racionalidad económica cuya base se fundamenta en la gestión de los servicios ambientales que solo usufructúan la naturaleza para satisfacer las necesidades de la especie humana como especie dominante. Esta visión se ha globalizado efectuando un crecimiento económico sin límites en el cual se valora el patrimonio natural en el corto plazo, sin tener en cuenta que dicho patrimonio es finito y, por tanto, sus posibilidades de uso son limitadas en el futuro. La racionalidad económica asigna un valor económico a todos los seres del mundo, lo que se establece como la fuente o la raíz de la crisis ambiental, ante lo cual se establece que esta crisis es una crisis de conocimiento (Leff, 2008).

El tránsito a la sustentabilidad debe darse mediante valores y no solo por objetivos materiales, para lo cual se hace necesario desarrollar una ética del proceso que sea coherente con sus fines morales, es decir, si los fines son valores, los medios no son neutros, lo que hace necesario establecer una ética de los medios y los procedimientos para que estos sean coherentes con los fines (Leff, 2002).

De acuerdo con Ángel y Ángel (2002, p. 25) la ética ambiental se pregunta por los principios que deben regir un nuevo comportamiento hacia lo ambiental que signifique procesos de transformación antrópica, acordes con los potenciales ecológicos. aunque esto tiene dificultades en la praxis, debido a que el hombre no

En lengua inglesa sustainable se utiliza tanto sustentabilidad como para sostenibilidad en el sentido aquí descritos. 
puede ajustarse a las leyes ecosistémicas de resiliencia, puesto que el crecimiento y densificación como especie son proporcionales al modelo tecnológico empleado para encontrar recursos para satisfacer sus necesidades. Debido a esto, Ángel (2003, p. 256) plantea al respecto la necesidad de crear nuevos equilibrios ecosistémicos que permitan la permanencia de la vida, lo cual no implica el desarrollo sostenible sino establecer la cultura como estrategia adaptativa, y ello genera la necesidad de profundas transformaciones culturales.

Esas consideraciones culturales y ecosistémicas hacen que la praxis de la gestión ambiental precise de una visión ambiental compleja para tratar de comprender la realidad de manera holística; es así como se establecen cinco formas de ver las partes de la realidad: profunda y de manera amplia; con referencia a un deber ser estético y ético; ver las interrelaciones reales actuales y prever las posibles; verlas en dinámica como parte de procesos temporales que implican corto, mediano y largo plazo; verlas con respeto a sus propios intereses en el espacio y el tiempo (Carrizosa, 2000, p. 31).

La ética de la cultura moderna reduce la dinámica de la vida a un mundo en el cual solo el respeto es posible en las relaciones humanas, cuyas creencias hacen que la razón se establezca como única forma de relación ética; así, otras formas de vida quedan excluidas de dicha ética antropocéntrica. En este sentido, una ética incluyente se configura como una ética-estética, en el marco en que las culturas se expresan en un mundo de vida simbólico biótico y las estructuras de la vida, sus sistemas complejos de interrelaciones, se auto-crean de manera continua. Ello permite que la ética ambiental incluya otros interlocutores: los ecosistemas, la Tierra, el universo, sistemas a partir de los cuales se es emergencia, y de los cuales se sigue siendo parte. La especie humana no debería excluirse y considerarse culpable sino que debe ser educada para que comprenda el contexto en el cual está inmersa (Noguera, 2004, p. 59).

\section{GESTIÓN, AMBIENTALISMO Y ALTERNATIVAS}

La gestión ambiental es consecuente con variadas corrientes político-ideológicas cuyas posturas pueden agruparse así: un primer grupo de planteamientos que asumen cambios radicales de comportamiento humano frente a la naturaleza o frente al modelo de desarrollo y el segundo que presenta esquemas de gestión laxos, que son posibles en un modelo de desarrollo que hace uso funcional de la naturaleza para continuar con los procesos de crecimiento económico.

En el primer grupo se encuentra la corriente conservacionista, orientada a la protección de áreas de fauna y flora, y a la consolidación de las mismas como sitios 
de reserva para dichas especies, las cuales tienen derecho de existir, por estar presentes en el planeta Tierra, es decir, por su valor intrínseco, el cual debe ser respetado por el ser humano, mediante la implementación de medidas de control natal para disminuir la superpoblación que amenaza con destruir los ecosistemas, por lo que se promueve que los seres humanos se adapten a los ecosistemas y la necesidad de defender los derechos legales de la naturaleza (Sánchez, 2013).

En ese mismo grupo, la corriente humanística-crítica considera una gestión ambiental teniendo como punto de partida la satisfacción de las necesidades de la sociedad. Esta corriente contiene dos subcorrientes: el ecodesarrollo y el Modelo Mundial Latinoamericano. El primero formula alternativas tecnológicas, de planificación, educación y acciones jurídicas para unificar conservación y desarrollo. La segunda tiene fundamentos marxistas, y plantea la necesidad de cambiar el modelo de desarrollo actual por otro mediante el cual se planteen nuevas relaciones de producción que permitan una forma diferente de interactuar con la naturaleza (Pierri, 2005, p. 12).

En la actualidad, el segundo grupo está representado por el ambientalismo moderado. Esta es la corriente hegemónica en el ámbito mundial con su propuesta de desarrollo sostenible, a partir de la cual la gestión ambiental está supeditada. El desarrollo sostenible es considerado por la Constitución Política de Colombia de 1991 como un propósito nacional y señala, en primer lugar, que no podemos legar a las nuevas generaciones las condiciones de atraso, exclusión y pobreza actuales, y en segundo lugar, que el cambio en el modelo no es responsabilidad exclusiva del Estado, sino de todos los colombianos (Ministerio del Medio Ambiente, 1998, p. 4).

Sin embargo, pese a las similitudes entre la corriente humanística-crítica y el ambientalismo moderado en lo referido a la satisfacción de las necesidades humanas, la primera corriente plantea la necesidad de transformaciones al modelo de desarrollo, por el contrario, la segunda acepta el modelo de desarrollo basado en el crecimiento económico y lo propone como condición para hacer posible la gestión ambiental. De allí que tenga respaldo en el ámbito internacional por parte de diversas instancias políticas y económicas; sin embargo, esta última corriente presenta contradicciones profundas que conducen a que se presenten ambigüedades en la gestión ambiental. Dichas contradicciones generan cuestionamientos como: ¿qué tan factible es la gestión ambiental con un modelo de desarrollo que requiere un alto consumo de naturaleza para mantenerse?

En el ambientalismo moderado las prioridades son los seres humanos y mantener el modelo de desarrollo, por lo tanto, cualquier acción en contra de 
la naturaleza se justifica a partir de dichas prioridades. Ello genera un continuo deterioro del patrimonio natural, debido a las permanentes demandas políticas y económicas para expandir el suelo urbano sin consideraciones ambientales, entre otras demandas, producto de las mismas ambigüedades de la gestión ambiental, que hacen necesaria la presencia permanente de agentes que controlen la intensidad de los impactos ambientales, puesto que la misma estructura conceptual con la que fue concebida dicha gestión no permite que se generen cambios culturales que propendan por una transformación en el sistema de creencias de la sociedad que haga posible nuevas formas de relacionarse el ser humano en la naturaleza. De allí que existan otras maneras de concebir la gestión ambiental a partir de cambios culturales.

Es así como la gestión ambiental desde la sustentabilidad trasciende el conservacionismo para reconocer la urgencia de satisfacer las necesidades del ser humano sin desconocer potenciales ecológicos de la naturaleza; por lo tanto, dicha gestión ambiental, según este enfoque, reconoce las cosmovisiones de la sociedad con relación a la naturaleza, al igual que considera la apertura cultural hacia la diversidad y la diferencia para hacer posible el respeto por las distintas formas o estéticas presentes en la biosfera (Sánchez, 2013, p. 27).

La gestión ambiental desde la ética ambiental presenta algunos elementos comunes a la corriente humanística-crítica en cuanto a la necesidad de igualdad humana que haga posible renacer, de manera paulatina, sensibilidades hacia la naturaleza. Asimismo, comprende una transformación de las estructuras simbólicas de la cultura que implica un diálogo de saberes en el cual está presente el reconocimiento del yo y del otro (Noguera, 2004). Dicha gestión ambiental se aproxima a los aportes de la ecología profunda en cuanto al respeto por la naturaleza, la cual posee un valor intrínseco que difiere del valor económico al asignarle a las demás especies, el respeto por la vida más que supeditar su existencia a la satisfacción de las necesidades humanas.

La gestión ambiental desde la complejidad, planteada por Carrizosa (2000, p. 33), implica la necesidad de una visión compleja que identifique en los territorios todas las interrelaciones presentes entre sociedad y naturaleza. Esto aplica en todos los aspectos territoriales como la consideración de la movilidad de las especies naturales, al igual que la movilidad del ser humano, esto es, no perder de vista los corredores ambientales ni tampoco las necesidades sociales. Es decir, al tener una visión holística, las decisiones territoriales no solo se fundamentan en una prioridad sino en múltiples factores que envuelven el sentido de la vida y de la existencia en sus múltiples formas. 


\section{A MANERA DE COMENTARIO}

Las corrientes conservacionista y humanística-crítica se plantean como radicales en cuanto a sus propuestas que limitan las actuaciones del ser humano, en el caso de la primera, y cuestionan el modelo de desarrollo basado en el crecimiento económico, en el caso de la segunda. En consonancia, han tenido poca acogida en el ámbito internacional, dada la prevalencia de la economía convencional y la visión antropocéntrica de los discursos universales sobre desarrollo sostenible. Por otra parte, el ambientalismo moderado se constituye como la corriente hegemónica para la gestión ambiental puesto que establece el crecimiento económico como elemento fundamental para el logro de la conservación y/o cuidado de la naturaleza.

El asunto no es menor, si se tiene en cuenta que la hegemonía del crecimiento económico asociado con el desarrollo y progreso tiene en su base la transformación de bienes y servicios ambientales subsidiados por la naturaleza, la cual tiene capacidades limitadas de reproducción y sus tiempos de revitalización son diferentes a los de la recurrencia de las necesidades humanas. Así, gestionar desde el ambientalismo moderado es, en buena medida, universalizar mayor ingreso como meta de la sociedad, lo que deja de lado otras visiones de la relación entre humanos y no-humanos y otras ideas de progreso, evolución (coevolución), y desarrollo, y elimina la posibilidad de construir otros fines para la sociedad (solidaridad, justicia).

La sustentabilidad y la ética ambiental, aquí descritas, se consideran como una opción diferente, al plantear la necesidad de una racionalidad ambiental fundamentada en los potenciales ecológicos de la naturaleza, la valoración ética y estética, las culturas adaptativas, y la visión ambiental compleja; ello constituye, más que un modelo de gestión, una re-construcción de la mirada de la naturaleza como recurso (factor productivo), al tiempo que es una alternativa en la forma de construir esa mirada; en otras palabras, en más una construcción metaconceptual que la búsqueda de un método para articular sociedad y naturaleza para crecer. Es una forma de trascender la visión utilitarista y mercantilista del ambientalismo moderado que, sin embargo, presenta limitaciones por cuanto es cada grupo humano con sus relaciones (entre ellos y con los no-humanos) el que define su rumbo (sus fines) y la manera de cómo conseguirlos dónde se incluyen las relaciones con el medio físico.

Reconocer que existen formas diferentes de conocer la relación entre humanos y no-humanos (pensamiento sistémico, complejidad, holismo), y alternativas al análisis, así como el determinismo de la Modernidad plantea retos para quienes deciden avanzar en esa lógica, por cuanto ello implica la integración de saberes y los trabajos inter-transdisciplinares que también están en construcción en cuanto a formas y métodos. Esa configuración de emergencia meta-teórica (cómo construir, 
justificar y emplear el conocimiento generado) y meta-metodológica (cuál debe ser el método del pensamiento complejo) pudieran explicar la no consolidación de la gestión ambiental desde (y para) la sustentabilidad.

\section{BIBLIOGRAFÍA}

Agudelo, N. (2004). Lo que no dice el Desarrollo Sostenible: aproximación a una lectura Latinoamericana. En: Luna Azul.v.19, 1-10.

Ángel, A. (2003). La diosa Némesis: Desarrollo Sostenible o Cambio Cultural (Vol. 2). Cali: Corporación Universitaria Autónoma de Occidente. 407 pp.

Ángel, A., \& Ángel, F. (2002). La ética de la Tierra. Ética y medio ambiente. En: E. Leff, Ética, vida, sustentabilidad. Primera ed. 12-26. México: PNUMA. Red de Formación Ambiental para América Latina y el Caribe.

Carrizosa, J. (2000). ¿Qué es el Ambientalismo? La Visión Ambiental Compleja. Santafé de Bogotá: CEREC. Universidad Nacional de Colombia. PNUMA. 132 pp.

CEPAL. (1994). Economía y Ecología: dos ciencias y una responsabilidad frente a la naturaleza. División de Recursos Naturales y Energía. Recuperado el 03 de agosto de 2012, de IV Simposio Internacional de Turismo, Ecología y Municipio: http://www.eclac.cl/publicaciones/xml/8/19768/ lcr1457s.pdf

Cortés, R., \& Aguilera, M. (2012). Política Ambiental en Colombia entre la formulación y la implementación. En: Criterios N.․ 5, 111-128.

Ferry, L. (1992). La Ecología Profunda. En: Vuelta N. ․ 192, 31-43.

Leff, E. (2002). Ética por la Vida. En E. Leff (coord).Ética, Vida, Sustentabilidad. Primera ed., 288-314. México; Ministerio del Medio Ambiente. PNUMA. PNUD. 332 pp.

Leff, E. (2008). Discursos Sustentables. México: Siglo XXI Editores. 272 pp.

Ministerio del Medio Ambiente. (1998). Lineamientos para la Política Nacional de Ordenamiento Ambiental del Territorio. Bogotá: Ministerio del Medio Ambiente. 68 pp.

Noguera, A. P. (2004). El reencantamiento del mundo. Manizales: PNUMA. Universidad Nacional de Colombia. 205 pp.

Pierri, N. (2005). Historia del concepto del desarrollo sustentable. En: G. Foladori, \& N. Pierri (Edits.), ¿Sustentabilidad? Desacuerdos sobre el desarrollo sustentable. 27-80. México: Miguel Ángel Porrúa. UAZ. Cámara de Diputados LIX Legislatura. 223 pp.

Sánchez, D. M. (2013). Propuesta de gestión ambiental para el sistema de espacio público natural urbano de la conurbación Pereira Dosquebradas. Pereira: Universidad Católica de Pereira. 64 pp. 